(Aus dem Kaiserin Auguste Viktoria-Haus, Reichsanstalt zur Bekämpfung der. Säuglingssterblichkeit [Direktor: Prof. Langstein].)

\title{
Zur Prüfung der Frauenmilehverfälschung.
}

Von

\author{
F. Edelstein.
}

(Eingegangen am 9. August 1921.)

Den eigentlichen AnIaß zur nachstehenden Untersuchung bildeten von mir unternommene Versuche, die bereits bestehenden typischen Reaktionen für Frauenmilch- und Kuhmilch zu einer Differentialprobe zu verwerten, mit der man imstande wäre, in einfacher und halbwegs einwandfreier Weise eine Verfälschung der Frauenmilch festzustellen. Praktisch kommen für eine Verfälschung nur Kuhmilch und Wasser in Betracht. Aber während sich eine Verwässerung mit physikalischen Methoden, z. B. durch Bestimmung des Brechungsexponenten des Serums oder auf eine andere Weise relativ leicht nachweisen läßt, ist der Nachweis des Milchzusatzes nicht gerade einfach. Die sichersten Methoden zur Erkennung eines Zusatzes von Milch anderer Tierart sind die biologischen, die Präcipitinreaktion, die anaphylaktische Methode, die Methode der Komplementbildung. So schön auch diese Methoden an sich sind, so komplizieren sie doch sehr den Betrieb, beanspruchen viel Zeit und sind vor allem wegen des jetzt so teuren Tiermaterials zu kostspielig. Die anderen bekannten chemischen Methoden, die Umikoffsche, die Neutralrotreaktion nach Moro, die Tugendreichsche Reaktion mit Silbernitrat und die Bauersche Methode mit Nilblausulfat sind leider zur Erkennung einer Verfälschung nicht geeignet, sie fallen nicht eindeutig und zuverlässig genug aus und dienen ja eigentlich nur zur bloßen Unterscheidung von Kuh- und Frauenmilch. Selbst für diesen Zweck versagt die sonst so einfache und schöne Reaktion von Bauer $\mathrm{ab}$ und zu. So habe ich nicht selten beobachten können, da $B$ frische Frauenmileh, die mit Nilblausulfat keine Färbung geben sollte, einen trüben, schmutzig-grau-grünen Farbenton lieferte. Hübscher vielleicht und gleichmäßiger in der Farbennuance fiel die Reaktion aus, wenn ich statt Nilblausulfat Toluidinblau anwandte, indem ich 
mich in der sonstigen Ausführung an die Originalvorschrift von Bauer hielt. Mit Kuhmilch erhält man ein helles, mit Frauenmilch ein tiefes Blau. Bei Mischungen von $\mathrm{Kuh}$ - und Frauenmilch ist aber die Unterscheidung der Farbentöne schon viel schwieriger.

Meine Versuche setzten nun damit ein, daß ich mich zunächst bemühte, die Umikoff sche Reaktion zu verfeinern. Letztere besteht bekanntlich darin, daß sich Frauenmilch durch Zusatz von Ammoniak und Erhitzen auf eine Optimaltemperatur von $60 \%$ rotviolett. bzw. dunkelviolett färbt, Kuhmilch dagegen eine hellgelbliche bis bräunliche Farbe annimmt oder auch farblos bleibt. Die Unterscheidung der Farbentöne ist zwar ohne weiteres möglich, man erhält jedoch durch die gelblichweiße Eigenfarbe der Milch oft Mischfarben, die namentlich, wenn Mischungen von Kuh- und Frauenmilch vorliegen, die Entscheidung sehr erschweren können. Zur Erklärung der U mikoffschen Reaktion wird angenommen, daß das Zustandekommen der Farbe, deren Intensität sich je nach Konzentration des Ammoniaks abstufen läßt, mit der Entstehung von Zersetzungs- und Verharzungsprodukten zusammenhängt, die bei der Behandlung von Mono- und Disacchariden mit Alkalien auftreten. Vielleicht ist an der Reaktion auch die Zitronensäure beteiligt, im übrigen ist über das Wesen der U mikoff schen Reaktion im großen und ganzen nichts sicheres bekannt. Das eine scheint aber gewiß, daß es der größere Milchzuckergehalt ist, der in der Frauenmilch die dunklere Farbe bei Erhitzung mit Alkalien hervorruft. Da sich nun sowohl der Milchzucker als auch die Citronensäure bzw. ihre Salze in der Milch in hochdispersem Zustande befinden, so sagte ich mir, daß es gelingen müßte, in einem von den Eiweißbestandteilen befreiten Serum die Farbe schärfer und deutlicher hervortreten zu lassen. Diese Erwartung hat sich nicht erfüllt. Ioh erhielt manchmal klarere Farbennuancen, aber keineswegs immer. Die diesbezüglichen Versuche führten. zu keinem greifbaren Resultat. Auf keinen Fall bot die Enteiweißung erhebliche Vorteile. Ich griff nun auf die Tatsache des hohen Milchzuckergehaltes in der Frauenmileh zurück und versuchte den Farbenumschlag in Gegenwart irgendeines der üblichen Metallsalze bei der Reduktion der Lactose in Serum zur Ausführung einer Reaktion heranzuziehen. Da der Farbenveränderung ein bekannter chemischer Vorgang zugrunde liegt, war zu erwarten, da $\$$ sich der Verlauf der Reaktion leichter übersehen und leiten läßt. Die gewöhnliche Trommersche bzw. Fehlingsche Probe ist hierfür nicht geeignet. Verwendet man z. B. vom Fehlingschen Reagens Mengen von etwa $20 \mathrm{ccm}$ zu einer Menge Laktoserum, die etwa $\mathbf{1}-\mathbf{2} \mathrm{ccm}$ Milch entspricht, so erhält man überhaupt keine Farbendifferenz. Sowohl bei der Kuhmilch als bei der Frauenmilch bleibt die Farbe gleich blau. Aber selbst wenn man entsprechend kleinere Quantitäten des Reagens verwendet, so daß ein 
Umschlag zustande kommt, dann stört die Erkennung der Farbenunterschiede das ausgeschiedene und suspendierte rote Kupferoxydul, das sich bei Luftzutritt sehr leicht oxydiert und die Flüssigkeit wieder stärker bläut. In Betracht kam also nur ein Reagens, welches das ausgeschiedene Kupferoxydul in eine farblose bzw. weißgefärbte Verbindung überführt. Sehr geeignet für diesen Zweck ist die Bangsche Kupfersulfatlösung, die Bang zur quantitativen Zuckerbestimmung benutzt hatte. Diese Lösung enthält neben Kupfersulfat, Kaliumcarbonat und Kaliumbicarbonat noch Sulfocyankali, welches sich mit dem bei der Reduktion der Zuckerlösung ausscheidenden Kupferoxydul zu weißem Kupferrohdanür umsetzt. Mit der Bangschen Lösung ist es mir in der Tat gelungen, recht schöne Farbenabstufungen im Milchserum zu erzielen. Während bei reiner Kuhmilch die schöne blaue bis grünlichblaue Farbe bestehen bleibt, zeigt das Serum der reinen Frauenmilch, entsprechend seiner stärkeren Reduktionskraft bei der gleichen Konzentration eine gelbe bis bräunliche Farbe. Mischungen von Kuh- und Frauenmilch liefern Übergangsfarben von blau zu gelb, Zusätze von 20, 30 und auch von 10\% Kuhmilch zu Frauenmilch sind an den im ganzen helleren gelblich- bis bläulichgrünen Farbentönen kenntlich.

Zum Enteiweißen habe ich dialysiertes Eisenoxyd benutzt. Die Reaktion wird folgendermaßen ausgeführt. In einem $50 \mathrm{ccm} \mathrm{Meß-}$ kölbchen verdünnt man $10 \mathrm{ccm}$ Frauenmilch mit etwa $30 \mathrm{ccm}$ Wasser, tropft unter tüchtigem Umschütteln allmählich $4 \mathrm{ccm}$ der Eisenlösung hinëin, füllt bis zur Marke mit Wasser auf, schüttelt das ganze nochmal gründlich durch und filtriert durch ein trockenes Filter. Vom Filtrat werden $3 \mathrm{ccm}$ mit $20 \mathrm{ccm}$ Bangscher Lösung versetzt und die Mischung wird eine halbe Minute lang im Sieden erhalten. Die ursprüngliche tiefazurblaue Farbe der Lösung wandelt sich binnen kurzer Zeit in ein kräftiges Gelb bzw. Bräunlichgelb um. Bei der Kuhmilch genügen $4 \mathrm{ccm}$ zur Enteiweißung nicht. Wegen des höheren Eiweißgehaltes sind hier mindestens $8 \mathrm{ccm}$ notwendig, um ein klares Filtrat zu erreichen. Im übrigen stört ein trübes Filtrat die Reaktion nicht, nur ein Uberschuß von Eisen ist zu vermeiden, weil dann das Filtrat gelb wird. Man kann sogar die Probe direkt ohne Enteiweißung ausführen, indem man zum Beispiel 6 Tropfen Milch mit 3 Tropfen Wasser und $10 \mathrm{ccm}$ Bangscher Lösung vermischt und eine halbe Minute aufkocht. Der Unterschied der beiden Milcharten ist deutlich $z u$ sehen, die Kuhmilch nimmt eine blaue, die Frauenmilch eine gelbe bis weißlichgelbe Farbe an. Aber die Farbentöne sind verschwommen, daher ziehe ich die Ausführung in der enteiweißten Milch vor. Die Bangsche Lösung wird nach Originalvorschrift in der Weise zubereitet, daß man $25 \mathrm{~g}$ Kaliumcarbonat, $5 \mathrm{~g}$ Kaliumbicarbonat und $20 \mathrm{~g}$ Rhodankali in etwa $60 \mathrm{ccm}$ Wasser 
unter Erwärmen löst und dann nach dem Abkühlen eine Lösung von $1,25 \mathrm{~g}$ Kupfersulfat in $10 \mathrm{ccm}$ Wasser zusetzt, auf 100 auffüllt einige Stunden stehen läßt und filtriert. Man stellt sich am besten gleich größere Mengen her, die Lösung ist mindestens 3 Monate haltbar. Die bei der Reduktion entstehenden Farbentöne sind erst nach einiger Zeit maßgebend und zwar wenn die Mischung abgekühlt ist. Nach einigen Stunden kommt es auch hier zu einer Reoxydation.

Oben habe ich von gleicher Konzentration gesprochen. Diese ist natürlich unerläßlich, denn nur unter dieser Bedingung hat die Reaktion überhaupt einen Unterscheidungswert. Nun wird ja durch Auffüllen auf das gleiche Volumen dieser Forderung entsprochen. Aber bei kritischer Betrachtung doch nur scheinbar.

1. Verwende ich, um klare Filtrate zu erhalten, bei Kuhmilch z. B.9 und bei Frauenmilch $4 \mathrm{ccm}$ Eisenoxyd und fülle auf 50 auf, so habe ich zwar in beiden Fällen dasselbe Volumen, da aber bei Kuhmilch der Eiweißniederschlag viel größer ist, so ist die Filtratmenge und folglich auch die Konzentration der beiden Filtrate nicht die gleiche. Der Fehler ist zwar nicht sehr groß, doch nicht ohne weiteres zu vernachlässigen. Man kann übrigens, wenn man auf ein klares Filtrat verzichtet, sowohl bei Kuh- als bei Frauenmilch $4 \mathrm{ccm}$ Eisenoxyd anwenden und so den Fehler vermindern.

2. Die Konzentration ist ferner wesentlich abhängig von dem wechselnden Milchzuckergehalt. Es ist klar, daß je nach dem Zuckergehalt der Milch die Intensität der Farbe variieren wird. Liegt zufällig eine verhäItnismäßig zuckerarme Frauenmilch vor, dann wird die Reaktion einen Farbenton aufweisen, der mehr dem bläulichen der Kuhmilch entspricht. Mit diesem wechselnden und nicht zu beeinflussenden Faktor muß also gerechnet werden. Nun ist aber die Differenz zwischen dem Milchzuckergehalt der Kuhmilch und der Frauenmilch doch noch immer groß genug $(4-5 \% \mathrm{zu} 6-7 \%)$, so daß die Reaktion auch nach dieser Richtung hin für reine Kuhmilch und reine Frauenmilch keine wesentliche Einschränkung erleidet.

Ich habe im ganzen etwa 170 verschiedene Proben von Frauenmilch und etwa ebensoviele Proben von Kuhmilch untersucht und, von sehr seltenen Ausnahmen abgesehen, die Reaktion immer eindeutig gefunden. Weit schwieriger gestaltet sich die Entscheidung, wenn FrauenmilchKuhmilch oder Frauenmilch Wassermischungen vorliegen und die Reaktion zur Erkennung von Frauenmilchverfälschungen dienen soll. $Z$ war war in der weitaus überwiegenden Anzahl der von mir untersuchten Fälle eine Abstufung deutlich zu sehen und eine Fetsstellung der Verfälschung möglich, doch zeigt schon ein flüchtiger Blick auf die beiden Farbenskalen, wie vorsichtig man bei der Beurteilung vorgehen muß. 


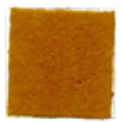

F.

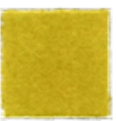

F.

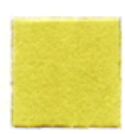

$20 \%$ KM.

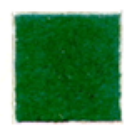

$30 \%$ KM.

Farbenskala I.

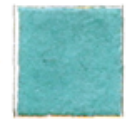

$20 \% \mathrm{~W}$.

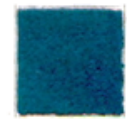

$30 \%$ W.

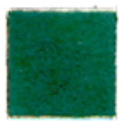

$20 \%$ KM. u. $10 \% \mathrm{~W}$.

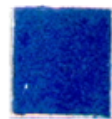

K.

Farbenskala II.

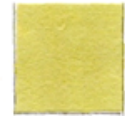

$20 \%$ KM.

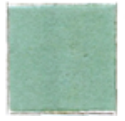

$20 \%$ W.

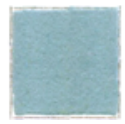

$80 \% \mathrm{~W}$.

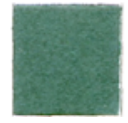

$20 \%$ KM. พ. $10^{\circ \% \mathrm{~W} .}$

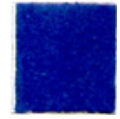

K.

F. bedeutet reine Frauenmilch, K. reine Kuhmilch, W. Wasserzusatz, KM. Kuhmilchzusatz.

Die Farbenskalen sind so entstanden, daß ich mir, von zwei verschiedenen willkürlich gewählten Frauenmilchproben ausgehend, entsprechende Mischungen mit Kuhmilch, Wasser und Kuhmilch und Wasser zusammenstellte, diese Mischungen nach der oben angegebenen Methode mit möglichst gleichen Mengen Fisenoxyd enteiweißte and vom Filtrat je $3 \mathrm{ccm}$ der Reduktion unterwarf. Innerhalb der ersten halben Stunde nach dem Kochen wurden die Farben mit Tusche zu Papier gebracht.*) Zwei für unsere Frage wichtige Tatsachen sind aus den Farbenskalen zu ersehen. Erstens, daß neben dem Kuhmilchzusatz der Wasserzusatz, wie ja zu erwarten war, durch die Konzentrationsverminderung die Farbentöne stark beeinflußt und zweitens, daß es von dem Grundton der bei der Reduktion des Frauenmilchserums entstehenden Farbe abhängt, wie die übrigen Nuancen ausfallen. Selbst wenn also eine Verwässerung in einer Probe sicher auszuschließen wäre, würde nur ein Vergleich mit dem Farbenton der reinen Frauenmilch, die im gegebenen Falle verfälscht vorliegt, ein sicheres Urteil erlauben. Das ist aber unmöglich. Man sieht also, daß, während es relativ leicht ist in einer Farbenreihe von der reinen Frauenmilch ausgehend die Farbenabstufungen gut abzuschätzen, die Beurteilung einer isoliert vorliegenden Mischfarbe große Utbung erfordert und nicht immer zuverlässig ist.

Ich habe deshalb, um diese Unsicherheit auszuschalten, noch eine Funktion, wenn ich. so sagen darf, eingeführt, und zwar den Eiweiß-

*) dus technischen Gründen ließen sich die mit Tusche aufgetragenen Original-Farbentöne nicht vervielfältigen. Vielmehr mußten für die Farbenskalen bereits im Handel vorhandene Farbenpapiere, wie sie der Verlag Unesma nach Anordnung von Geheimrat $\mathrm{O}$ stw ald herstellt (siehe $\mathrm{O}$ st wald s Farbenfibel), verwendet werden. Es ist ferner zu berïcksichtigen, daß die Farbentöne im Reagensglas bzw. im durchscheinenden Licht anders anssehen als auf mattem Papier. Deshalb halte ich es für zweckmäßiger, daß man sich zur Orientierung seine Farbenskala selbst herstellt. 
gehalt der betreffenden Milch. Die Differenz zwischen dem Eiweißgehalt der Frauenmilch und der Kuhmilch ist so groß, daß sie noch günstiger für unsere Zwecke $z u$ verwerten ist, als die Differenz im Zuckergehalt. Es bedarf dazu keiner langwierigen Analyse, ich bediene mich zur Erkennung des Eiweißgehaltes eben der Fällungsreaktion mit Eisenoxyd, mit dem ich die Enteiweißung vornehme. Die Farbe des Filtrats gibt mir eine schnelle Antwort. Man muß nur wissen, welche

Tabelle I. Fraueninileh.

$\mathrm{tr}=$ trübe, Eliweiß noch nicht vollkommen gefällt; $\mathrm{o}=\mathrm{klar}$, Eiweiß gefällt; $g=g e l b$, Übersehuß von Eiweils.

\begin{tabular}{|c|c|c|c|c|c|c|c|c|c|c|c|c|c|}
\hline \multirow{2}{*}{ ن } & \multicolumn{12}{|c|}{ Menge des zu $10 \mathrm{ccm}$ Milch zugesetzten Lisenoxyds } & \multirow{2}{*}{$\begin{array}{l}\text { Redultions- } \\
\text { farbe }\end{array}$} \\
\hline & $1, \mathbf{5}$ & 1,75 & 2 & $2, \overline{5}$ & 3 & $\mathbf{3 , \mathbf { 5 }}$ & 4 & 4,5 & s & $\mathbf{5 , 0}$ & 6 & 4,5 & \\
\hline 1. & - & - & - & & - &.- & $\mathrm{O}$ & o & 0 & $g$ & $\underline{g}$ & $\cdots$ & - \\
\hline 2. & - & - & $\mathrm{O}$ & - & $\cdots$ & & - & $\stackrel{r}{r}$ & $g$ & 一 & - & & $\cdots$ \\
\hline 3. & - & - & t & $\operatorname{tr}$ & 0 & & -- & - & - & $g$ & $g$ & & \\
\hline 4. & - & tr & - & & - & , & $\mathrm{O}$ & $g$ & - & $\stackrel{c r}{r}$ & 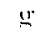 & -- & \\
\hline 5. & - & - & - & & - & -- & - & -- & - & $\stackrel{r}{r}$ & - & & $\cdots-$ \\
\hline 6. & -- & - & $\cdots-$ & $\ldots \ldots$ & - & & $\ldots$ & $\ldots$ & & $g$ & -- & - & -- \\
\hline 7. & - & $\ldots$ & - & $\cdots$ & $\ldots$ & & - & & $\ldots$ & $\mathrm{g}$ & & . & --- \\
\hline 8. & $\cdot-$ & -- & $\ldots$ & -- & $\cdots$ & & $\cdots$ & -- & $\cdots$ & o & - & & $\ldots$ \\
\hline 9. & - & - & $\cdots$ & &.. & & -.- & - &.$- \cdot$ & $g$ & $\cdots$ & & $\cdots$ \\
\hline 10. & - & - & $\cdots$ & & $\cdots$ & & -. & - & -- & $\cong$ & $\cdots$ & $\cdots$ & \\
\hline 11. & - & -- & - & 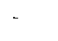 & & & $\cdots$ & -- - & & $\underline{\prime \prime}$ & & & - \\
\hline 12. & - & - & $\cdots$ & $\operatorname{tr}$ & $\operatorname{tr}$ & o & - & & -- & o & 0 & $\underline{g}$ & $\cdots$ \\
\hline 13. & -- & $\cdots$ & - & o & 0 & -- & $\cdots$ & $\cdots$ & $\cdots$ & $\stackrel{r r}{r}$ & $\cdots-$ & - & $\cdots$ \\
\hline 14. & - & - & - & tr & o & & $-\cdot$ & - & $\cdots$ & o & & $\cdots$ & \\
\hline 15. & - & - & $\cdots-$ & - & -- & $\cdots$ & --- & --- & -- & o & - & $\cdots$ & - \\
\hline 16. & - & - & - & & & & $\cdots$ & $\cdots$ & - & o & 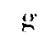 & -- & \\
\hline 17. & - & 一 & $\operatorname{tr}$ & o & & & $\cdot-$ & $\cdots$ & $\cdots$ & $\stackrel{r}{r}$ & $\cdots$ & $\ldots \ldots$. & $\cdots$ \\
\hline 18. & - & -- & $\operatorname{tr}$ & $\mathrm{tr}$ & tr & 0 & -- & -- & -- & 0 & $g$ & & \\
\hline 19. & - & $\cdots-$ & tr & tr & tr & tr & o & - & -- & o & $\underline{r}$ & - & \\
\hline 20. & - & $\cdots$ & - & - & - & o & - & -- & $g$ & - & - & -- & welb \\
\hline 21. & - & - & & $\cdots$ & $\ldots$ & . & 0 & 0 & $\mathrm{~g}$ & -- & - & $\cdots$ & bräunl.-gelb \\
\hline 22. & - & $\cdots$ & & $\operatorname{tr}$ & & & 0 & - & 0 & $g$ & & 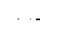 & bram \\
\hline 23. & - & . & - & $\operatorname{tr}$ & $\mathrm{tl}^{\prime}$ & & o & -- & $\cdots$ & $g$ & $\cdots$ & $-\cdot$ & $"$ \\
\hline 24. & - & & $\cdots$ & $\operatorname{tr}$ & $\cdots$ & . & o & - & ....- & $g$ & & $\cdots$ & $"$ \\
\hline 25. & - & - & tr & - & - & & - & & o & $\underline{g}$ & & & $"$ \\
\hline 26. & - & tr & - & $-\cdot$ & $-\cdots$ & $\cdots$ & o & $\cdots$ & $g$ & $\cdots$ & & -- & $n$ \\
\hline 27. & - & -- & $\operatorname{tr}$ & - & & & $\cdots$ & $-\cdot$ & o & tr & - & & $"$ \\
\hline 28. & - & $\ldots$ & $\operatorname{tr}$ & & . & - & - & - & o & $\underline{y}$ & $\cdots$ & & $n$ \\
\hline 29. & - & -- & - & $\cdots-$ & - & - & 0 & o & $g$ & --- & . & $\cdots$ & bräunt.-pell) \\
\hline 30. & - & -. & $\operatorname{tr}$ & & $\cdots$ & - & o & - & o & - & -- & - & $"$ \\
\hline 31. & - & $\operatorname{tr}$ & o & $\cdots \cdots$ & $\cdots \cdots$ & - & o & $g$ & - & - & -- & $\cdots-$ & dunkel-brituı \\
\hline 32. & - & - & $\operatorname{tr}$ & $\operatorname{tr}$ & $\cdots$ & - & 0 & $g$ & - & $\cdots$ & $\cdots$ & $\cdots$ & bräunl.gelb \\
\hline
\end{tabular}


F. Edelstein :

Tabelle II. Kuhmilch.

\begin{tabular}{|c|c|c|c|c|c|c|c|c|c|c|c|c|c|c|c|}
\hline \multirow{2}{*}{$\begin{array}{l}\text { 崩 } \\
\text { 总宽 }\end{array}$} & \multicolumn{14}{|c|}{ Menge des zu $10 \mathrm{~cm}$ Miloh zugesetzten Eisenoxyds } & \multirow{2}{*}{$\begin{array}{l}\text { Reduktions. } \\
\text { farbe }\end{array}$} \\
\hline & 5 & 6 & 7 & 8 & 9 & 10 & 18 & 28 & 29 & 80 & 31 & 32 & 83 & 84 & \\
\hline 1. & - & - & - & - & - & $\ldots$ & - & 0 & 0 & $\mathrm{~g}$ & - & - & - & - & - \\
\hline 2. & - & - & - & 一 & - & - & - & - & - & $\ldots$ & - & $g$ & - & - & - \\
\hline 3. & - & - & - & - & -- & - & - & - & - & - & - & 0 & 0 & - & - \\
\hline 4. & - & - & - & - & - & - & - & - & - & - & - & 0 & 0 & - & - \\
\hline 5. & -- & - & - & - & - & 0 & - & - & - & - & - & - & - & $\mathrm{g}$ & - \\
\hline 6. & - & - & - & - & - & 0 & - & - & - & -- & - & - & - & $\mathrm{g}$ & - \\
\hline 7. & $\mathrm{tr}$ & $\operatorname{tr}$ & $\operatorname{tr}$ & 0 & $\dot{-}$ & - & - & - & - & - & - & $g$ & $\mathrm{~g}$ & - & - \\
\hline 8. & $\operatorname{tr}$ & tr & $\operatorname{tr}$ & $\operatorname{tr}$ & $\operatorname{tr}$ & o & - & - & - & $\cdots$ & $\mathrm{g}$ & $g$ & - & - & - \\
\hline 9. & -- & $\operatorname{tr}$ & o & $\mathrm{O}$ & - & - & - & - & - & $\mathbf{g}$ & - & - & - & - & $\ldots$ \\
\hline 10. & -- & - & $\operatorname{tr}$ & 0 & - & - & - & - & - & - & $\mathrm{g}$ & - & - & - & - \\
\hline 1. & - & - & - & $\operatorname{tr}$ & 0 & 0 & - & - & - & - & - & - & - & - & - \\
\hline 2. & - & - & -- & - & $\operatorname{tr}$ & 0 & - & - & - & $\ldots$ & $\ldots$ & - & -- & - & - \\
\hline 13. & -- & -- & - & $\operatorname{tr}$ & $o$ & - & o & - & $\mathrm{g}$ & -- & - & - & - & - & - \\
\hline 4 & - & - & - & - & $\operatorname{tr}$ & 0 & - & - & - & $\mathrm{g}$ & - & - & - & - & - \\
\hline 15. & - & - & - & - & - & o & - & - & - & - & - & - & - & - & - \\
\hline b. & - & $\operatorname{tr}$ & - & - & 0 & - & o & - & - & $\mathrm{g}$ & - & - & -. & - & - \\
\hline 7. & & - & $\ldots$ & $\operatorname{tr}$ & 0 & - & - & - & $g$ & $\mathrm{~g}$ & - & - & - & - & - \\
\hline
\end{tabular}

Tabelle ILL. Frauenmilchmit $20 \% \mathrm{Kuhmilch}$ verfälscht.

\begin{tabular}{|c|c|c|c|c|c|c|c|c|c|c|c|c|c|c|c|c|c|c|c|}
\hline \multirow{2}{*}{ 总这 } & \multicolumn{18}{|c|}{ Menge des zugesetzten dialysierten Eisenoxyds in $\mathrm{ccm}$. } & \multirow{2}{*}{$\begin{array}{l}\text { Reduktions- } \\
\text { farbe }\end{array}$} \\
\hline & 2 & 2,5 & 8 & $\overline{0}$ & 4 & 4,5 & ह &, 5 & 6 & 6,5 & 7 & 7,5 & 8 & $3, \mathbf{5}$ & 9 & 9,5 & 10 & 10,0 & \\
\hline 1. & - & - & $\operatorname{tr}$ & $\operatorname{tr}$ & 0 & 0 & 0 & - & - & - & - & 0 & $\mathrm{~g}$ & $g$ & $\mathrm{~g}$ & -1 & - & - & - \\
\hline 2. & L & - & $\operatorname{tr}$ & 0 & - & 一 & - & o & - & - & - & $\mathrm{O}$ & $g$ & 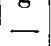 & $\begin{array}{l}c \\
g\end{array}$ & -1 & - & - & $\ldots$ \\
\hline 3. & - & $\ldots$ & $1-$ & $\operatorname{tr}$ & 0 & - & - & - & $\ldots$ & - & - & - & O & $\mathrm{g}$ & - & - & - & - & 一 \\
\hline 4. & $\operatorname{tr}$ & 0 & - & - & $\mathrm{g}$ & _ & 1. & - & - & - & - & - & - & - & - & - & - & - & - \\
\hline 5 & - & tr & - & - & $\stackrel{-}{-}$ & - & - & - & - & o & - & - & 0 & - & - & - & - & - & gelb \\
\hline 6. & - & $\operatorname{tr}$ & - & - & - & - & - & $-\ldots$ & - & - & - & -. & o : & - & $g$ & - & - & - & \\
\hline 7. & - & $\operatorname{tr}$ & - & - & - & 一 & - & -- & - & - & - & - & o & - & $\mathrm{g}$ & - & - & - & \\
\hline 8. & -- & - & - & $\operatorname{tr}$ & - & $\mathrm{O}$ & 0 & - & - & - & - & - & - & - & - & - & - & - & grünlich-gelb \\
\hline 9. & - & - & - & $\operatorname{tr}$ & o & - & — & - & - & 一 & - & - & o & - & - & -1 & - & - & bläa \\
\hline 0 & - & - & - & $\boldsymbol{-}$ & tr & 0 & - & - & - & - & - & - & $-i$ & $\mathrm{~g}$ & - & - & - & - & $g r$ \\
\hline 1. & - & - & tr & - & $o$ & - & - & - & $\ldots$ & - & - & - & - & $g$ & - & - & - & - & grünlich-gelb \\
\hline 2 & - & - & - & tr & o & o & - & - & - & - & - & - & — & - & - & - & - & - & gel \\
\hline F & - & - & - & $\operatorname{tr}$ & 0 & 0 & - & - & - & - & - & - & -1 & - & - & - & - & - & \\
\hline 4. & - & - & $\operatorname{tr}$ & o & 0 & - & - & - & - & - & - & - & - & - & - & -1 & $1-$ & - & \\
\hline b. & - & - & - & $\operatorname{tr}$ & 0 & 0 & - & - & - & - & - & - & -1 & - & - & - & - & - & 0 \\
\hline 6. & - & - & - & tr & 0 & - & -1 & - & - & - & - & - & $--\mid$ & - & - & $g$ & - & - & \\
\hline$\pi$ & - & $\operatorname{tr}$ & 0 & - & $\mathrm{g}$ & - & - & - & - & - & - & - & - & - & - & - & - & - & gelblich-g \\
\hline 8. & - & - & - & $\operatorname{tr}$ & 0 & 一 & $\ldots$ & - & - & - & - & - & 0 & - & - & $g$ & - & - & 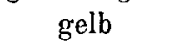 \\
\hline 0 & - & - & $\operatorname{tr}$ & ) & - & - & - & - & _- & - & - & - & o & $\mathrm{g}$ & - & - & -1 & - & gelb \\
\hline
\end{tabular}

Zeichenerklärung: $\operatorname{tr}=$ trübe, Eiweiß noch nicht vollkommen gefällt; $o=k l a r$, Eiweiß gefällt; $g=$ gelb, Überschuß von Eisenoxyd. 
Tabelle IV. Frauenmilch mit $30 \%$ Kuhmilch verfalscht. $\operatorname{tr}=$ trübe, Eiweiß noch nicht vollkommen gefällt; $o=k l a r$, Eiweiß gefällt; $\mathrm{g}=\mathrm{gelb}$, Überschuß von Eiweiß.

\begin{tabular}{|c|c|c|c|c|c|c|c|c|c|c|c|c|c|c|c|c|c|c|c|c|}
\hline \multirow{2}{*}{ 总芶 } & \multicolumn{19}{|c|}{ Menge des zugesetzten dialysierten Eisenoxyds in ccm } & \multirow{2}{*}{$\begin{array}{l}\text { Redultions- } \\
\text { farbe }\end{array}$} \\
\hline & 2 & 2,6 & 3 & 3,5 & 4 & 4,5 & 5 & 5,5 & 6 & 0,5 & 7 & 7,5 & 8 & 8,5 & 9 & 9,5 & $10 \mid$ & 10,5 & 11 & \\
\hline 1. & - & - & $\operatorname{tr}$ & - & $\ldots$ & 0 & 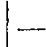 & o & - & - & - & - & - & - & 0 & -1 & $\mathrm{~g}$ & - & 1 & 一 \\
\hline 2. & - & - & tr & 一 & o & - & - & o & - & - & - & - & - & o & $\mathrm{g}$ & - & - & - & - & - \\
\hline 0. & - & - & - & - & $\operatorname{tr}$ & o & 一 & - & - & - & - & - & - & o & $g$ & - & - & - & - & $-\infty$ \\
\hline 4. & $\operatorname{tr}$ & o & - & $\dot{-}$ & 0 & - & $\mathrm{g}$ & - & - & - & - & - & - & - & - & - & - & - & - & - \\
\hline 5. & - & - & tr & - & - & 一 & - & - & 一 & -+ & - & - & 0 & - & - & - & $g$ & - & - & grünlich-gelb \\
\hline U. & - & tr & 一 & - & - & - & - & - & - & - & - & - & 0 & - & -1 & - & $\mathrm{g}$ & - & - & " \\
\hline 7. & - & $\operatorname{tr}$ & - & - & - & - & - & - & - & - & - & - & - & 0 & - & $g$ & $g$ & $1 \div$ & - & \\
\hline 8. & - & - & - & - & - & - & $\operatorname{tr}$ & O & - & - & - & $\longrightarrow$ & - & - & 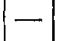 & - & - & - & - & grún \\
\hline 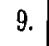 & -1 & - & 一 & - & - & tr & - & o & - & - & - & - & - & - & - & $g$ & - & - & - & bläulich-grun \\
\hline 0. & - & - & - & - & 一 & — & tr & o & - & 一 & - & - & - & - & $g$ & - & - & - & - & grün \\
\hline 1. & -1 & - & $\rightarrow$ & tr & 一 & o & - & - & - & - & - & - & - & - & $g$ & - & - & $\div$ & - & \\
\hline 2. & -1 & - & - & - & - & tr & - & o & - & - & - & - & - & - & - & $\mathrm{g}$ & - & - & - & \\
\hline 3. & - & - & -- & - & - & tro & o & - & - & - & - & - & - & - & o & 一 & - & - & - & grünlich-gelb \\
\hline 4. & - & - & - & - & $\operatorname{tr}$ & - & - & 0 & - & $\longrightarrow$ & - & - & - & - & - & $\mathrm{g}$ & - & - & - & \\
\hline 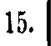 & - & - & - & - & tr & - & o & - & -- & - & - & - & - & - & - & o & - & - & - & grun \\
\hline 16. & -1 & - & - & - & $\operatorname{tr}$ & - & o & - & - & - & - & - & - & - & - & - & - & - & $g$ & 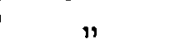 \\
\hline 17. & - & - & tr & o & o & 0 & - & - & - & - & - & - & - & - & - & - & - & - & - & bläulich-grün \\
\hline 18. & - & - & tr & o & - & 一 & - & - & - & - & - & - & - & - & - & - & $g$ & $一$ & - & ge \\
\hline & -1 & - & $1-$ & lis & $1-$ & $\mathrm{o}$ & $1-$ & $1-$ & $1-$ & -1 & 1 - & - & $1-1$ & $1-$ & $1-1$ & 10 & $g$ & $|-|$ & $1-1$ & grelb-grün \\
\hline
\end{tabular}

Mengen des Fällungsmittels nötig sind, um gerade einen Úberschuß zu erzielen. Ist diese Abgrenzung bei Frauenmilch, Kuhmilch und bei Frauenmilch-Kuhmilch-Mischungen vorgenommen, dann läßt sich aus der Menge des zugesetzten Eisenoxyds, aus der gelben Farbe des Filtrats und unter Zuhilfenahme der Reduktionsfarbe die Verfälschung feststellen.

Die vorstehenden Tabellen zeigen die maximalen Mengen des dialysierten Eisenoxyds an, welche erforderlich sind, um in $10 \mathrm{ccm}$ Frauenmilch, Kuhmileh usw. das Eiweiß quantitativ zu fällen bzw. eben einen therschuß zu erzeugen. Für Frauenmilch (Tabelle I) liegt die Höchstgrenze zwischen 5 und 6, in weitem Abstande davon bewegt sich die Grenze für Kuhmilch (Tabelle II) zwischen 29 und 34, für die mit 20 bzw. 30\% Kuhmilch vermischte Frauenmilch (Tabelle III und IV) beträgt die Maximalmenge 9-10 und für die Frauenmilch-Wassermischung (Tabelle V) liegt die Grenze zwischen 3 und 4. Allerdings sind diese Grenzen keine feststehenden, sie schwanken $a b$ und zu nach oben und unten. So gibt es z. B. Kuhmilchproben, die über $35 \mathrm{ccm}$ Eisenoxyd*) pro $10 \mathrm{ccm}$ Milch zur quantitativen Eiweißausfällung

*) Die Eisenlösung (Ferrum oxydatum dialysatum liquid. Me r ck bzw. Liquor ferri oxychlorati dialysati des D. A.B. V.) soll ein spezifisches Gewicht von 
bzw. bis zum Überschuß verbrauchen. Eine derartige Milch würde, zur Frauenmilch zugesetzt, die Fällungsgrenze des Gemisches über 10 hinaus verschieben. Vor einem Irrtum schützt aber in solchen Fällen die Reduktionsfarbe.

Der Gang der Untersuchungen würde sich nun folgendermaßen abspielen: Je $10 \mathrm{ccm}$ der vorliegenden Milch verdünnt man in einem 50-ccm-Meßkölbchen mit $30 \mathrm{ccm}$ Wasser, fügt, am besten aus einer Bürette, tropfenweise und unter Umschütteln Eisenoxyd hinzu, füllt bis zur Marke auf und filtriert durch ein trockenes Filter. Man fängt z. B. mit $3 \mathrm{ccm}$ an, fügt das zweitemal 6 , das dritte $8 \mathrm{~cm}$ hinzu und sieht zu, welche Menge an Eisen ein gelbes Filtrat liefert. Von derjenigen Probe; die bis zur Erzielung eines klaren bzw. gering getrübten

TabelleV. Frauenmilch mit $20 \%$ bezw. $30 \%$ Wasser. tr $=$ trube, Eiweiß noch nicht rollkommen gefällt; $o=k$ lar, Eiweil gefällt; $\mathrm{g}=$ gelb; Überschuß von Eisenoxyd.

\begin{tabular}{|c|c|c|c|c|c|c|c|c|c|}
\hline \multirow{2}{*}{ ن } & \multicolumn{8}{|c|}{ Menge des zugesetzten dialysierten Eisenoxyds in com } & \multirow{2}{*}{$\begin{array}{l}\text { Reduktions- } \\
\text { farbe }\end{array}$} \\
\hline & 1 & 1,5 & 2 & $2, \overline{0}$ & 3 & 3,5 & 4 & $4, \tilde{s}$ & \\
\hline 1. & - & $\operatorname{tr}$ & - & 一 & o & - & $\mathrm{g}$ & - & - \\
\hline 2. & - & $\mathrm{o}$ & 一 & $\cdots$ & -- & - & o & $g$ & blau-grun \\
\hline 3. & $\operatorname{tr}$ & - & - & - & o & $g$ & 一 & - & grünlich - blau \\
\hline 4. & o & - & - & 一 & o & - & $\mathrm{g}$ & 一 & grün \\
\hline 5. & 0 & - & - & - & o & $\mathrm{x}$ & - & - & grünlich - blau \\
\hline 6. & $t_{1}$ & 0 & - & - & 一 & - & $\mathrm{g}$ & - & grülich - blau \\
\hline 7. & $\operatorname{tr}$ & 0 & $\ldots$ & - & - & - & $g$ & $\dot{-}$ & $\sim$ \\
\hline 8. & - & - & - & 0 & $\mathrm{~g}$ & -- & - & $\dot{-}$ & blaul \\
\hline 9. & o & - & - & - & o & - & $g$ & - & gruinlich-blau \\
\hline 10. & 0 & - & $\mathrm{o}$ & - & - & - & - & - & blau \\
\hline
\end{tabular}

Filtrates am wenigsten Eisen verbrauchte, wird mit $3 \mathrm{ccm}$ des Filtrats die Reduktionsprobe ausgeführt und die Reduktionsfarbe festgestellt.

Das Resultat ergibt sich aus der Tabelle VI. Diese ist allerdings kurz schematisch zusammengestellt und enthält nur die typischen Verfälschungsgruppen. Andere Verfälschungsformen lassen sich nicht schwer aus der Tabelle herausfinden. So wird sich bei einer Verfälschung der Frauenmilch mit sehr wenig Wasser und viel Kuhmilch die Reduktions-

1,043-1,047 und einen Eisengehalt von 3,3-3,6\% Fe aufweisen. Das Präparat muB unter gutem VerschluB und unter genïgendem Schutz gegen Tageslicht, und Sonne an einem kühlen Ort aufbewahrt werden. Aher selbst unter Einhaltung dieser Vorsichtsmalregeln tritt bei längerem Stehen eine Zersetzung bzw. Gehaltsverminderung an Fe ein. Deshalb ist dringend anzuraten, möglichst frische Lösungen $z u$ verwenden und sie nach Möglichkeit direkt von der Fabrik (Merck) zu beziehen. Genügen z. B. von einer älteren Eisenlösung für reine Kuhmilch nicht 40 und für reine Frauenmilch nicht $7 \mathrm{ccm}$ zum UbersohuB, dann verwerfe man sie lieber und verwende ein frisches Präparat. 
farbe mehr der unter Nr. 2 angegebenen und schließlich der der reinen Kuhmilch (blau) nähern, während die Menge des bis zum Überschu $B$ anzuwendenden Eisenoxyds der unter Nr. 2 verzeichneten entsprechen wird. Liegt dagegen lediglich eine Verwässerung vor, dann wird zwar die Reduktionsfarbe grünlich-blau ausfallen, scheinbar also auf eine Kuhmilch hinweisen, aber die Eisenoxydmenge wird wegen des verminderten Eiweißgehaltes unter 4 sinken. Oder nehmen wir z. B. an,

Tabelle VI.

\begin{tabular}{|c|c|c|c|}
\hline 这 & \begin{tabular}{|c|} 
Anzahi der zugesetzten \\
ccm Eisenoxyd zu $10 \mathrm{ccm}$ \\
Mileh, bis das Filtrat \\
gelb gefärbt ist
\end{tabular} & Reduktionsfarbe & Resultat \\
\hline $\begin{array}{l}1 \\
2 \\
3\end{array}$ & $\begin{array}{c}5-6 \\
\text { uber } 9 \\
\text { unter } 4\end{array}$ & $\begin{array}{l}\text { gelb bis braun } \\
\text { grünlich-gelb b. gelbl.-grün } \\
\text { blau bis grünlich-blau }\end{array}$ & $\begin{array}{l}\text { reine Franenmilch } \\
\text { Kuhmilchzusatz } \\
\text { Wasserzusatz }\end{array}$ \\
\hline
\end{tabular}

daß von einer Milch je $10 \mathrm{ccm}$ mit $4 \mathrm{bzw} .5 \mathrm{bzw} .8 \mathrm{ccm}$ ein farbloses, mit 8,5 ein hellgelbes mit 9 ein deutlich gelbes Filtrat liefern, so kann das auf eine Kuhmilchverfälschung, aber daneben auch auf eine Verwässerung hindeuten. Wir führen nun mit $3 \mathrm{ccm}$ des Filtrates derjenigen Probe, die $4 \mathrm{ccm}$ Eisen verbraucht hatte eine Reduktion aus, die Reduktionsfarbe ist bläulich-grün. Also handelt es sich um eine mit Kuhmilch und Wasser verfälschte Milch. Bei reinem Kuhmilchzusatz wäre nämlich die Reduktionsfarbe gelblich-grün ausgefallen, der bläuliche Ton würde fehlen. Noch andere Modifikationen ließen sich natürlich anführen, ich begnüge mich aber mit der Andeutung. Von einer Ausprobierung ausgeklügelter, ,analysenfester " Verfälschungsformen habe ich abgesehen. Sie kommen kaum vor, wären übrigens durch geschicktes Interpolieren aufzudecken. Für den gewöhnlichen Gebrauch in Säuglingsheimen usw. wird die kombinierte Methode rasch und bequem zum Ziele führen.

\section{Zusammenfassung.}

Die Eigenschaft des hohen Milchzucker- und niedrigen Eiweißgehaltes der Frauenmilch wird zur Erkennung einer Frauenmilchverfälschung herangezogen. Aus dem Farbenton, der bei der Reduktion einer Kupferlösung durch den Milchzucker des Lactoserums entsteht, und aus der zur quantitativen Ausfällung des Eiweißes nötigen Menge Eisenoxyds wird geschlossen, ob und was für Verfälschung vorliegt.

\section{Literaturverzeichnis.}

1) Umik off, Jabrb. f. Kinderheilk. 42, 356. 1896. - ${ }^{2}$ ) M or o, Münch. med. Wochenzchr. 1912, S. 2553. - $\left.{ }^{3}\right)$ T u gend re i ch, B rl. klin. Wochenschr. 1911, S. 224. - 4) Ba uer, Monatschr. f. Kindrrheilk. 11, 474. 1913. - ${ }^{5}$ ) Bang, Bioch. Zeitschr. 2, 271. 1907; 11, 538. 1908 und 32, 443. 1911.

Charlottenburg, Kaiserin Auguste Viktoria-Haus. 